\title{
Are Vectors Able to Learn about their Hosts? A Case Study with Aedes aegypti Mosquitoes
}

\author{
Wladimir J Alonso ${ }^{+}$, Tristram D Wyatt, David W Kelly \\ Zoology Department, Oxford University, South Park Road, Oxford, OX1 3PS, England
}

The way in which vectors distribute themselves amongst their hosts has important epidemiological consequences. While the role played by active host choice is largely unquestioned, current knowledge relates mostly to the innate response of vectors towards stimuli signalling the presence or quality of their hosts. Many of those cues, however, can be unpredictable, and therefore prevent the incorporation of the appropriate response into the vector's behavioural repertoire unless some sort of associative learning is possible.

We performed a wide range of laboratory experiments to test the learning abilities of the mosquito, Aedes aegypti. Mosquitoes were exposed to choice procedures in (1) an olfactomenter and (2) a 'visual arena'. Our goal was to determine whether the mosquitoes were able to associate unconditional stimuli (blood feeding, human breath, vibration and electrical shock) with particular odours (citral, carvone, citronella oil and eugenol) and visual patterns (horizontal or vertical black bars) to which they had been previously observed to be responsive.

We found no evidence supporting the hypothesis that associative learning abilities are present in adult Ae. aegypti. We discuss the possibilities that the assays employed were either inappropriate or insufficient to detect associative learning, or that associative learning is not possible in this species.

Key words: Aedes aegypti - learning - insect vectors

The spatial distribution of a population of blood-sucking insects amongst its available vertebrate hosts has important epidemiological consequences for the transmission of vector-borne diseases. In fact, after daily survival rate, their vectorial capacity is most sensitive to changes in host preference (Dye \& Hasibeder 1986). Therefore, knowledge of the biological parameters that lead to host choice can be highly relevant for planning of disease control (McCall \& Kelly 2002).

Regardless of their individual previous experience, mosquitoes are attracted to their hosts based on several stimuli to which each species (and even populations or individuals) responds in a complex and idiosyncratic way (Cardew 1996, Clements 1999). Whereas a genetic basis for differences in host choice is a well documented phenomenon (e.g.: Gillies 1964), in addition, several authors (see below) have suggested also that experience could change some of their behavioural repertoire, such as their response to host and oviposition odours, and even might be involved in the spatial memorization of resources.

The consensus from studies looking for evidence of learning in mosquitoes remains equivocal. Some find no evidence for learning (Rawlings \& Curtis 1982, ArredondoJimenez et al. 1992). Others have obtained positive results. In some of them, their data do not strictly rule out

Financial support was received from Capes (BEX1521/98-5), Brazil.

${ }^{+}$Corresponding author. Fax: +44-1865.31044. E-mail: wladimir.alonso@zoo.ox.ac.uk

Received 26 August 2002

Accepted 7 May 2003 alternative explanations, such as orientation towards the closest pertinent chemical source (Ribbands 1949, Giglioli 1965, Renshaw et al. 1994), or that the heterogeneity of behaviour found in the population was an effect of the existence of genetic polymorphism (Hii et al. 1991). Charlwood et al. (1988) showed positive results that can only be attributed to learning of a memorized home range, nevertheless this work suffers from 'pseudoreplication' (Hurlbert 1984) owing to the intrinsic difficulties associated with a field study.

Recent works (Mwandawiro et al. 2000, McCall \& Eaton 2001, McCall et al. 2001) have been more conclusive. Mwandawiro et al. (2000) found in 3 Japanese Encephalitis vectors, that host loyalty to bloodfeed on cow or pigs was not passed onto offspring, indicating that this behaviour has a learning component, instead of a genetical one. In the work of McCall and Eaton (2001) odours presented during the larval/pupal stage were preferred by adults of Culex quinquefasciatus in choice of oviposition site. McCall et al. (2001) also showed in a fieldwork in Tanzania, how 30 from 44 Anopheles arabiensis individuals (recovered from over 17000 found in 2 different houses and released at a intermediate point) return to their original collection places.

Specifically in the case of the search for available hosts by mosquitoes and other blood-sucking insects, Kelly and Thompson (2000) propose that the ability to learn would be adaptive to individuals aiming to maximise their feeding success and minimise their chances of being killed in the process, to the extent that it would allow them to feed preferentially on the least defensive hosts. For instance, individual vectors could be more 'loyal' to a subgroup of host species or populations upon which they previously fed successfully. Here we performed a comprehensive series of positive and negative conditioning experiments based mainly (but not exclusively) on aspects 
that could be related to host choice behaviour, using the yellow fever and dengue vector mosquito Ae. aegypti as our laboratory model.

\section{MATERIALS AND METHODS}

Insects - Ae. aegypti individuals were taken from a laboratory culture present in the Zoology Department of Oxford University, which before reaching the UK belonged to the University of Notre Dame, Indiana (US), where it was established in the 1950's (Roger J. Wood, pers. commun.). The culture room was maintained at $26-29^{\circ} \mathrm{C}$ and $60-80 \%$ relative humidity, under a $12: 12 \mathrm{~h}$ light:dark cycle. Larvae were fed with dog biscuits and TetraMin tropical fish food. Adults were maintained in mixed-sex colonies (cages measuring $17 \times 17 \times 17 \mathrm{~cm}$ ) in cohorts of the same age, and provided with water alone until used in the experiments. Experimental adults were between 1 and 3 days old, and used only once. In the case of females, only those individuals that responded positively to human breath were used. Transport between cages and testing apparatus was carried out using a manual aspirator. In the experiments where required, warm defibrinated sheep's blood (TCS Microbiology Company ${ }^{\circledR}$ ) was provided through a membrane feeding system (Hemotek ${ }^{\circledR}$ ).

Experimental room - The experimental room temperature ranged between $24-27^{\circ} \mathrm{C}$. A single $40 \mathrm{~W}$ lamp placed $80 \mathrm{~cm}$ overhead illuminated each testing apparatus. A cylindrical netting curtain screen was connected from the lamp to the bench top, completely encircling the apparatus, so that an observer could monitor the behaviour of the subjects without disturbing them. An overhead video camera installed beside the light source recorded mosquito behaviour for later analysis.

All experiments were performed between 10:00 and 18:00 h. All materials were carefully handled with disposable gloves to prevent odour contamination, and the testing apparatus was cleaned with $99.7 \%$ ethyl alcohol after each replicate.

Experiments using odours as conditioned stimuli

An apparatus (Fig. 1) was built to test whether the mosquitoes could associate either punishment or reward (the positive or negative 'unconditioned stimuli', US, respectively) with 1 of 2 odours (the 'conditioned stimuli', CS). The odours were presented on filter papers in cartridges upwind of the Y-tube (Fig. 1D). In the training stage, odour A was presented without additional stimulus, and odour B was presented with an unconditioned stimulus (for details of each experiment, see below). In different replicates the position of the odours, the order of the association with the unconditioned stimuli (first or second), and the odour that was associated with the unconditioned stimulus was varied in a balanced design, to control for these variables in the subsequent analysis.

During the training stage the mosquitoes remained inside a plastic cartridge (the conditioning chamber: Fig. $1 \mathrm{H})$, and were exposed to odours A and B consecutively. In the 'testing stage', the two odours were presented simultaneously, each flowing down one arm of the Y-tube (Fig. 1F). A metal gauze gate (Fig. 1I) was opened, allowing the mosquitoes to move into the Y-tube.

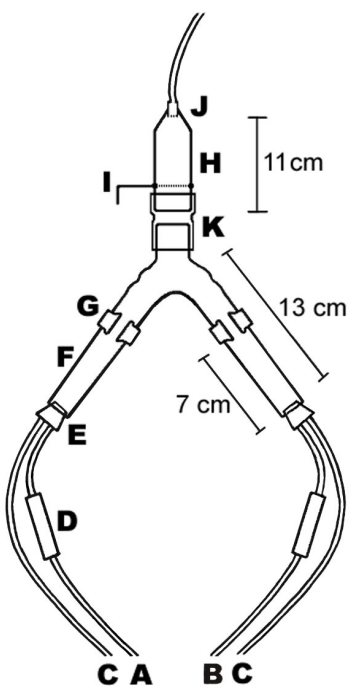

Fig. 1: diagram showing the apparatus used for testing whether Aedes aegypti mosquitoes could associate punishment or reward with particular odours. The humidified and filtered air (see text) is conducted in by a series of valves (not shown) as fresh air (C), or as odorised air in pipes A and B, after passing through the odour cartridges (D). Passing through the rubber stoppers (E) the air enters the arms of the $\mathrm{Y}$ tube $(\mathrm{F})$ that is attached to a table by a pair of clips (G). The mosquitoes' training vial (H) has a screen door (I) that allows the air to flux constantly, until it is expelled from its other extremity $(\mathrm{J})$ to outside the room. The door can be opened manually from outside to allow the mosquitoes to enter the $\mathrm{Y}$ tube. The manufacture of the connection $(\mathrm{K})$ between $\mathrm{H}$ and $\mathrm{F}$ varies depending upon the training procedure (see text).

The air that entered the apparatus was pushed with a oilless pump (Charles Austen Pumps, model F.65 DE), filtered with activated charcoal and humidified to approximately $80 \%$ RH. The airflow was controlled at $4 \mathrm{l} / \mathrm{min}$ so that a speed of about $4 \mathrm{~m} / \mathrm{min}$ in the exit end of the $Y$ tube was achieved. Air from the apparatus was expelled outside the room (Fig. 1J). The suitability of the apparatus was tested in preliminary trials by presenting individuals with a choice between a pure flux of air and a flux of air mixed with human breath, which showed that most individuals consistently preferred the latter arm of the Y-tube

Unless otherwise stated, in each experiment each series of trials consisted of 20 replicates for each sex, each replicate containing 20 individuals.

Vibration as negative unconditioned stimulus - Mechanical activity, in the form of skin twitching, stamping, slapping, etc., is one of the main cues that a mosquito can use to gather information about the defensiveness of a potential host (Edman \& Scott 1987, Clements 1999, Kelly 2001). We tested whether Ae. aegypti mosquitoes would be able to associate odours with vibration of the conditioning chamber by fixing the vial (Fig. $1 \mathrm{H})$ to a small aquarium pump (Tetra Whisper 400), which made it vibrate at 50 Hertz (amplitude: 1mm). This stimulus seems to have provided a significant level of disturbance for the individuals inside the vial, which kept taking off and trying to land on its walls most of the time unsuccessfully when the vibration was applied. In order to provide a flex- 
ible junction between the $\mathrm{Y}$ tube and the mosquitoes' vial (Fig. 1K) cut fingers of powder-free latex gloves were used. The odours presented as CS were $20 \mu \mathrm{l}$ of carvone (Aldrich 435759) or citral (Sigma C83007). These odorants were used since (1) in preliminary trials we observed that the mosquitoes were able to perceive them (avoiding them in upwind flight when they were presented separately in the $\mathrm{Y}$ tube in a choice with a fresh air option), and (2) because they are chemically very distinct, and this difference seems to be noticed by the mosquitoes given their unequal distribution in the $\mathrm{Y}$ tube - with a small tendency towards preferring the citral - when both were presented together. (Using the same training and testing procedure, but without the presentation of the CS - see methodology elsewhere. Number of replicates for each sex: 10 . Number of individuals per replicate $=20$. Results - female: citral mean $=5.1$ s.d. $=2.5$; carvone mean $=4.2$ s.d. $=1.8$; male: citral mean $=6.2 \mathrm{s.d}$. $=1.5$; carvone mean $=3.1 \mathrm{s.d}$. $=$ 1.5. Dependent variable $=$ mosquitoes in each arm after 1 $\min$ ).

The sequence of odour presentation for each set of mosquitoes was $30 \mathrm{~s}$ of one odour associated with the unconditioned stimuli and $30 \mathrm{~s}$ of the other one alone. Fifteen seconds of resting (with fresh air, Fig. 1C) were allowed in between the presentations of the odours. This procedure was performed 5 times before conducting the test stage. The same experiment was used to test females and males separately.

Breath as positive unconditioned stimulus - Human breath provides reliable information about a host presence and its position and strongly attracts Ae. aegypti (Kellogg \& Wright 1962). Odours associated with it could be used as additional cues to find a host. We therefore repeated the previous experiment but instead of providing vibration as a negative US, we provided human breath as a positive US.

As the junction between the $\mathrm{Y}$ tube and the mosquitoes' vial we used a hard rubber tube in which a metal pipe with small holes (for gas diffusion) was introduced. During the association period, human breath, blown continuously by the experimenter, was diffused (c.a. $0.5 \mathrm{l} /$ $\min$ ) into the chamber.

Blood feeding as positive unconditioned stimulus A novel odour associated with successful blood feeding could provide important information to be used by mosquitoes when seeking a subsequent blood meal. To test this, mosquitoes up to 3 days old were randomly distributed between two cages. On the following day, one of the cages (control) was transported to another room (same conditions of humidity and temperature), left undisturbed and provided with a source of blood for $70 \mathrm{~min}$. At the same time, an aquarium pump (Tetra Whisper 400) pushed a small flow of fresh air into a cartridge containing a blank filter paper and into the cage. Subsequently, another cage (treatment) was placed in the same room, in the same conditions (with new blood in the feeder), but with a filter paper containing $20 \mu \mathrm{l}$ of citral in the cartridge connected to the air pipe. In a previous experiment we observed that this amount of citral is the minimum quantity that the subjects could detect: they were significantly repelled by it when given a choice between this amount of citral and fresh air, something that did not occur when the amount of citral was only $2 \mu 1$.

Although most of the females had engorged with the blood provided, in the following day (3rd experimental day) the procedure was repeated with both experimental groups to allow a second opportunity for feeding, as Ae. aegypti females regularly feed on the second as well as the first day of the gonotrophic cycle (Canyon et al. 1999). On subsequent days sugar diluted in water $(1: 10)$ was made available.

Three and 5 days later (6th and 8th exp. days), after observing that a large number of eggs had already been laid, 20 replicates (each with 10 females) of each treatment were tested in the Y tube in a balanced way. The subjects were presented with choices between filter paper containing $20 \mu \mathrm{ml}$ of citral, and blank filter paper. The number of individuals in each arm was scored as stated for the previous experiment.

The difference in the number of individuals that chose the arm with the odour, against those choosing the control arm, was then compared between the control and the treatment groups. Only females were tested.

Electric shock as negative unconditioned stimulus Although a stimulus that is not usually found in nature by insects, electric shocks have proved to be a useful tool for investigating learning abilities in this class of organisms, notably in another dipteran species, Drosophila melanogaster. We adopted the method of Tully and Quinn (1985), for our apparatus. Our electrified grid was the same as that used in experiments with Drosophila (Quinn et al. 1974, Tully \& Quinn 1985). It consisted of a copper pattern printed on a 57 x $81 \mathrm{~mm}$ flexible epoxy backing, which was used to line the inside of the training vial. During the test stage a separate circular grid also covered the extremity of the training vial that opened towards the $\mathrm{Y}$ tube. Therefore, approximately $70 \%$ of the total surface of the training vial was lined with the electric grid. The possibility that some individuals remained during the whole experimental period in the non-electrified surface was lowered since many of the subjects were prompted to fly by the electrical pulses, disrupting the resting position of the others.

The electrical stimuli consisted of pulses of $120 \mathrm{~V}(50$ $\mathrm{Hz}$ ), lasting $1 \mathrm{~s}$ and given at intervals of $5 \mathrm{~s}$. This voltage was chosen as the lowest one in which we were able to observe reactions from the mosquitoes. Only one training cycle with electrical shock was applied to each group of mosquitoes, as employed in fruit fly studies.

In order to minimize the possibility of negative results as being consequence of the odours and quantities used, 2 distinct sets of experiments were conducted using the electric shock as US. In the first one, the odours and amounts were identical to those used in experiment A1 (citral $20 \mu \mathrm{l}$, carvone $20 \mu \mathrm{l}$ ). In the other, citronella oil (EHPNatural Product Division, Batch nr. SR1925) and eugenol (Sigma E5504) were employed, but this time the amount in the filter paper was $2 \mu \mathrm{l}$ : sufficient to promote an avoidance response by the mosquitoes when presented against fresh air into the Y tube. Only females were used. 
Analysis of the data - The response measures were obtained by analysing the video-tape records and scoring how many individuals were in each arm $1 \mathrm{~min}$ after the opening of the gate. Other periods were also registered ( $15 \mathrm{~s}, 30 \mathrm{~s}, 2 \mathrm{~min}, 3 \mathrm{~min}, 4 \mathrm{~min}$ and $5 \mathrm{~min}$ ) in some experiments, but 1 min was chosen because it was the shortest time after the training phase when the distribution of individuals between the arms of the Y tube could be considered well established (see Fig. 2). Only those subjects located at least $7 \mathrm{~cm}$ beyond the entrance of either arm of the $\mathrm{Y}$ tube were considered.

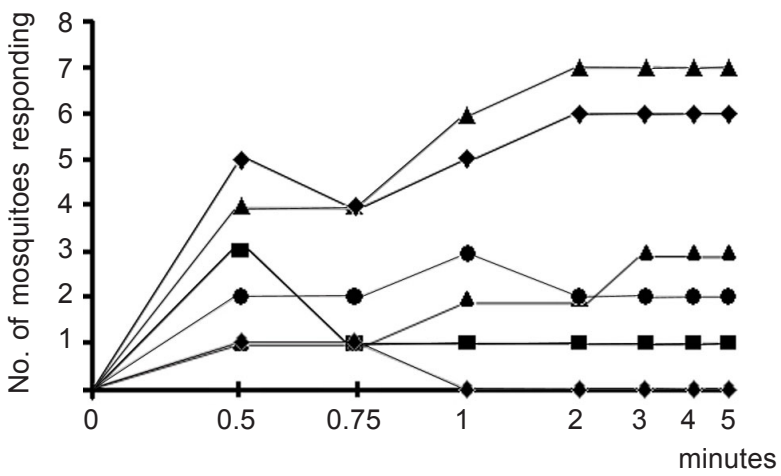

Fig. 2: examples (randomly chosen) of how the mosquitoes distribute between the two arms of the $\mathrm{Y}$ tube over a period of $5 \mathrm{~min}$ (intervals of $15 \mathrm{~s}, 30 \mathrm{~s}, 1 \mathrm{~min}, 2 \mathrm{~min}, 3 \mathrm{~min}, 4 \mathrm{~min}, 5 \mathrm{~min}$ ). Each line represents a different observation.

The non-parametric Wilcoxon matched-pairs test was performed separately for the replicates of males and females in each experiment. One-tailed tests were performed, as we predicted a priori that subjects would avoid the arm releasing the odour associated with the US when this was a punishment (vibration, electrical shock) and would choose preferentially the arm with the other odour. In those cases where the stimulus was either human breath or blood, we expected that the subjects would use the odour released simultaneously as a cue of host presence, and during the test they would move preferentially towards the odour associated to the CS. For all statistical tests, the alpha level was set to 0.05 .

Although the experiment was designed in a way so as to compensate for possible trends towards one of the sides of the apparatus and one of the odours, and the order in which the odours were presented, these variables were also analysed to investigate potential biases.

Experiments using a visual cue as conditioned stimuli

Here we tested the hypothesis that Ae. aegypti mosquitoes are able to search for their previous host (or host type) in order to resume an interrupted blood meal based on the memory of a visual pattern associated to a successful blood-meal received a few seconds before.

For this experiment, we set up a simple device where images could be presented and hidden, just by rotating a white bucket (internal bucket, 'IB') with 4 holes $(8.5 \mathrm{~cm}$ of diameter) in its walls, inside another white bucket (external bucket, 'EB') containing black images and patterns on its inner walls. A transparent plastic lid on IB allowed the illu- mination, observation and video-recording of the interior of the set-up. Both buckets (HK-Plastics EC 10000) had a capacity of 101 (c.a. $23 \mathrm{~cm}$ height and $26 \mathrm{~cm}$ diameter).

Each replicate, using a naive individual, consisted of: (a) the training stage, where a visual pattern (CS) was displayed on top of a feeding membrane offering warmed blood (US) at its centre, through a hole in the wall of the EB; (b) the testing stage, where 2 distinct patterns (positioned at a distance of $17 \mathrm{~cm}$, or $90^{\circ}$ of arc from each other) were revealed on the opposite side from the now concealed training image and feeder.

Upon release, each individual generally landed on the visual pattern for a few seconds, and from there - and probably guided by the warmth of the feeder - moved towards the centre of the image where they could blood feed. After allowing the individual to feed for $25 \mathrm{~s}$, its feeding was interrupted by a gentle rotation of the IB. This process was repeated 3 times for each individual. Ae. aegypti females take about $284 \pm 40 \mathrm{~s}$ to take their blood meal (Jones \& Pilitt 1973), and they will still try to complete their blood meal immediately if this is interrupted before a critical amount of blood is consumed. We chose three blood-feeding periods of $25 \mathrm{~s}$ because, after them, the mosquitoes were clearly prone to resume their host search at once. After training, the testing stage was introduced by rotating the IB in a way such that the training shape was hidden to the mosquito, which could now see, approach and land on the testing shapes.

The images were made of a black adhesive tape stripe $(8 \times 2.5 \mathrm{~cm}$ each), in one case placed vertically on the bucket's wall, and the other one horizontally. We anticipated that the mosquitoes could distinguish between these patterns in our experimental device. The eyes of adult Ae. aegypti have relatively poor resolution $\left(\approx 12^{\circ}\right.$, Muir et al. 1992a), but this should be enough to distinguish the visual cues (given their size, proximity and simplicity) we presented to them. As these mosquitoes are attracted mostly to stationary objects of low reflectance and solid colour (Muir et al. 1992a) we used only continuous black images to provide a maximum contrast over the white background, and because colour is not a stimulus to which they respond (Muir et al. 1992b). The experiment was blocked for the direction of the rotation of the bucket, the position of the images in the training stage, and the type of image used as US.

Analysis of the data - Video records of 41 replicates (out of 60) were considered for the final analysis. The other 19 were rejected, either because they were not clear enough or because the mosquito spent most of its time inactive or flying towards the light. Data records from the videotape were (blindly) analysed for $1 \mathrm{~min}$ from the onset of the testing stage. We predicted that, in the existence of preference towards the image on which they had fed before $(\mathrm{CS}+)$, the mosquitoes would approach that image and/or land on it in preference to the control one (CS-) in the following ways: (a) choosing it first after the presentation of the 2 options in the test stage; (b) presenting a shorter latency to choose it when they chose it first; (c) choosing it with a higher frequency; (d) once landed, remaining a longer time on it. 
An approach towards each image was scored if the individual entered into an area drawn around each target in front of the monitor screen. Landing was recorded when not only the individual touched the visual pattern, but also stopped flying and perched in the visual pattern surface. Several tests were employed depending upon the nature of data (see Results for details).

\section{RESULTS}

Experiments using odours as conditioned stimuli

Vibration as US - After 20 replicates the trends obtained for both sexes were an avoidance of the odour associated with punishment $(\mathrm{CS}+)$, as predicted. In males (Fig. 3) this trend was not significant $(\mathrm{p}=0.23)$. However, a significant trend $(p=0.02)$ was detected in females. As we had a high probability of obtaining positive results (learning in this species) by chance owing to the multiplicity of tests for the same hypothesis, we decided to double our sample size to 40 . At this point, the significance was not maintained $(\mathrm{p}=0.17)$ and we could not reject the null hypothesis that the higher proportion of mosquitoes in the 'escape' arm was obtained by chance. These results are summarized in Fig. 3.

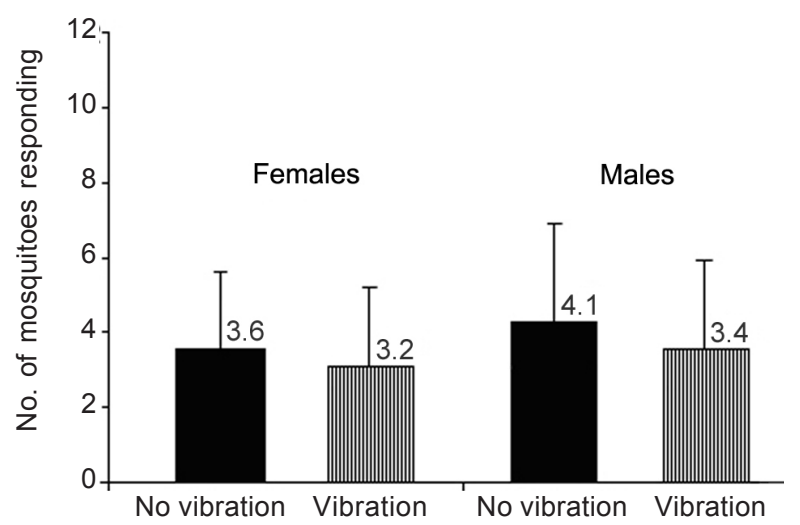

Fig. 3: mean number of mosquitoes in the arm that released the odour previously associated with vibration $1 \mathrm{~min}$ after the beginning of the test stage, versus the number of mosquitoes in the arm with the odour not associated with punishment. Females $n=40$ replicates, males $\mathrm{n}=20$ replicates. Each replicate comprised 20 individuals.

Human breath as US - There was no preference for breath as the CS (Fig. 4). In males, there was a slight tendency to approach, as predicted, the 'breath' arm, but this trend was not significant $(\mathrm{p}=0.43)$. Contrary to our expectations, females were more likely to choose the arm releasing the odour not associated with human breath.

Blood feeding as US - The proportion of mosquitoes that went to the citral stimulus did not differ between the groups that had been exposed to citral when blood feeding and those exposed to fresh air when blood feeding (Fig. 5). Statistical testing has not been applied in this experiment because all the control and all the treatment mosquitoes were trained separately, but all the mosquitoes in each treatment were trained together; therefore they were not independent.

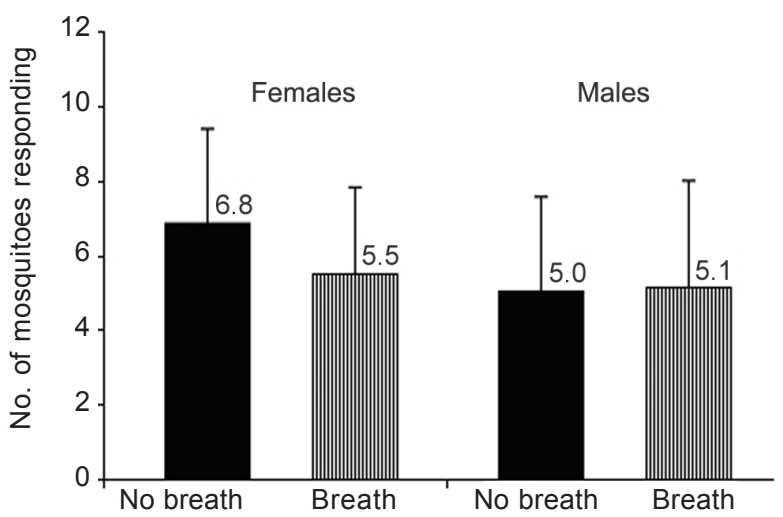

Fig. 4: mean number of mosquitoes in the arm that released the odour previously associated with breath $1 \mathrm{~min}$ after the beginning of the test stage as compared to the number of mosquitoes in the arm with the odour not associated with this reward. Females $n=19$ replicates, males $\mathrm{n}=20$ replicates. Each replicate comprised 20 individuals.

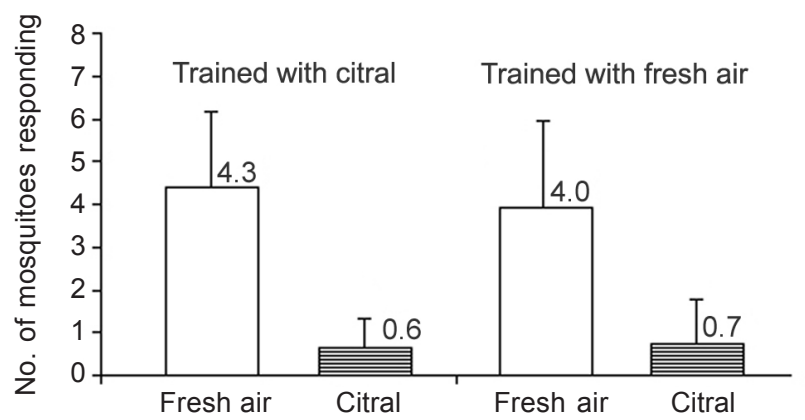

Fig. 5: responses to citral versus fresh air between mosquitoes submitted to exposition to citral and those submitted to only fresh air when blood feeding. Results are expressed as the mean number of individuals that $1 \mathrm{~min}$ after the beginning of the test stage were in each arm of the $\mathrm{Y}$ tube. $\mathrm{N}=20$ replicates for each treatment group (only females employed). Each replicate comprised 20 individuals.

Electric shock as $U S$ - When citral $(20 \mu \mathrm{l})$ and carvone $(20 \mu \mathrm{l})$ were employed as CS, a non-significant $(\mathrm{p}=0.09)$ trend towards avoiding the odour associated with electrical shock was obtained. This tendency was not maintained when citronella oil $(2 \mu \mathrm{l})$ and eugenol $(2 \mu \mathrm{l})$ were used instead (Fig. 6).

Applying Bonferroni correction (Sokal \& Rohlf 1995: 703) on the Y-tube data also does not allow the rejection of the null hypothesis, as the critical alpha level is divided by the number of tests (in this case $\mathrm{n}=7$ ), which give us a $\alpha^{\prime}=0.007$, a value smaller than the $p$-value $(p=0.02)$ we had initially obtained.

Experiments using a visual cue as conditioned stimuli

All mosquitoes included in the analysis $(\mathrm{n}=41)$ performed several approaches to both patterns (Table - c), and only two of them approached only one of the patterns. Most of the mosquitoes did not land on any of the visual patterns during the first minute of the test stage 


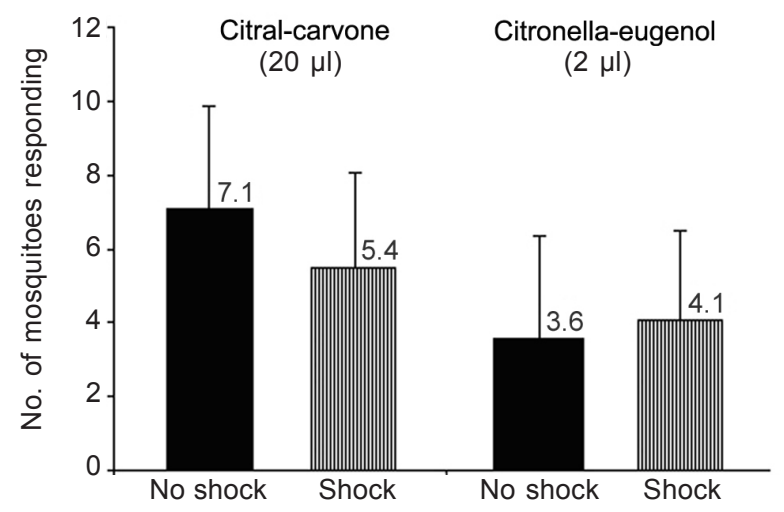

Fig. 6: mean number of mosquitoes in the arm releasing the odour previously associated with electric shock $1 \mathrm{~min}$ after the beginning of the test stage, versus the number of mosquitoes in the arm with the odour not associated with punishment. The odorants employed are indicated above the results of each comparison. $\mathrm{N}=20$ replicates for each treatment group (only females employed). Each replicate comprised 20 individuals.

(Table - c). Among those that did land, 12 individuals did it only on 1 of the patterns ( 7 on the CS+, and 5 on the CS-), 1 did it on both patterns, and finally 1 mosquito landed twice on the CS+ and once on the CS-.

Most mosquitoes approached and landed first on the image where they had been previously blood-fed. Nevertheless, these trends were not significant (Table - a). However, they did not approach the CS+ more quickly (in fact, the trend was in the opposite direction, Table - b).

The time taken to the first landing was less for the CS+ as expected, but again it was not significantly different from the time taken to land on the CS-. Landing frequency was higher for the CS+ (Table - d), but not significantly different from that for the CS-.

Therefore, overall, these results do not provide us with unequivocal evidence for the existence of a preference for the visual pattern on which subjects were partially blood-fed previously.

\section{DISCUSSION}

There can be little doubt about the basic assumption that mosquitoes have a neurological in-built equivalent to that found in Drosophila, where learning and memory skills had been extensively described (Waddell \& Quinn 2001). Their phylogenetic proximity and the neuro-anatomical descriptions of mosquitoes support this hypothesis. Therefore, finding learning ability in mosquitoes would not be surprising (McCall \& Eaton 2001).

However the aim of our research was to find learning abilities that could make adaptive sense to the problems that a mosquito faces in nature, such as blood-feeding. Certainly the odours we employed were not the ones that this species would naturally use to locate hosts, but we purposely chose odours that were detectable by our mosquitoes, but were not innately attractive, as would be the case for e.g. $\mathrm{CO}_{2}$, acetone, lactic acid or 1-octen-3-ol (Cardew 1996, Clements 1999). We did so because we predicted that, in order to detect an olfactory cue that could be used to 'qualify' a sub-set of hosts during the lifespan of a mosquito, individuals could not rely on features that are shared by all hosts or do not change between mosquito generations. Instead, they should rely on very particular odours (like those derived from the food ingested by the host) that would influence in an idiosyncratic way the general odour profile of the host.

Visual patterns would also be expected to be useful for allowing an individual mosquito to find a previous host and resume an interrupted blood feeding. Experiments in a flight simulator (Ernst \& Heisenberg 1999) suggested that Drosophila could be conditioned to discriminate vertical and horizontal bars when a heat source (a beam of infrared light) was used as punishment during conditioning. Making the assumption (quite basic, given the simplicity of the choices and the data currently available for this species, e.g. Muir et al. 1992a,b, Clements 1999) that our experimental mosquitoes too can distinguish both patterns, our results do not support the hypothesis that they can use this information to return to a previous host. In any case, if they are not able to rely on the presence of

TABLE

Parameters and statistics relative to the data obtained in the first minute of the test stage. 'CS+' refers to the image that had been previously associated, for 'conditioning' purposes, with blood-feeding. 'CS-' refers to the image that had not been previously associated to blood-feeding. When pertinent, descriptive statistics are shown as mean \pm standard deviation. One-tailed tests are summarised below each pair data. When the direction of the results is the expected, one-tailed statistics are showed below the descriptive data, otherwise only n.e.d. (non-expected direction) is shown

\begin{tabular}{|c|c|c|c|c|c|c|c|c|}
\hline & \multicolumn{2}{|c|}{$\begin{array}{c}{ }^{\mathrm{a}} \text { First choice } \\
\text { (number of mosquitoes) }\end{array}$} & \multicolumn{2}{|c|}{$\begin{array}{c}\text { b Time until } \\
\text { 'First choice' (seconds) }\end{array}$} & \multicolumn{2}{|c|}{${ }^{\mathrm{c}}$ Occurrence } & \multicolumn{2}{|c|}{${ }^{\mathrm{d}}$ Duration (seconds) } \\
\hline & $\mathrm{CS}+$ & CS- & $\mathrm{CS}+$ & CS- & $\mathrm{CS}+$ & CS- & $\mathrm{CS}+$ & CS- \\
\hline \multirow[t]{2}{*}{ Landing } & 9 & 5 & $23.1 \pm 16.1$ & $38.5 \pm 15.3$ & $0.24 \pm 0.49$ & $0.17 \pm 0.38$ & $28.7 \pm 25.1$ & $18.6 \pm 13.8$ \\
\hline & \multicolumn{2}{|c|}{$\begin{array}{c}\text { Binomial test } \\
p=0.21\end{array}$} & \multicolumn{2}{|c|}{$\begin{array}{c}\text { Mann-Whitney U } \\
\text { 1-tailed } \\
\mathrm{p}=0.06\end{array}$} & \multicolumn{2}{|c|}{$\begin{array}{l}\text { Wilcoxon S. Ranks } \\
\text { 1-tailed } \\
p=0.20\end{array}$} & \multirow{2}{*}{\multicolumn{2}{|c|}{$\begin{array}{c}\text { Mann-Whitney U } \\
\text { 1-tailed } \\
\text { p }=0.34\end{array}$}} \\
\hline \multirow[t]{2}{*}{ Approach } & 22 & 19 & $11.8 \pm 6.5$ & $9.1 \pm 7.2$ & $8.1 \pm 4.5$ & $8.5 \pm 5.7$ & & \\
\hline & \multicolumn{2}{|c|}{$\begin{array}{c}\text { Binomial test } \\
p=0.38\end{array}$} & \multicolumn{2}{|c|}{ n.e.d. } & \multicolumn{2}{|c|}{ n.e.d. } & & \\
\hline
\end{tabular}


these very different patterns, we tentatively conclude that Ae. aegypti individuals would also not be able to rely on other, more subtle, visual differences in nature.

In the series of experiments described, we used a range of unconditioned stimuli as 'punishment', from vibration, which would be also found in natural conditions, to a more severe stimulus - electric shock, routinely and successfully employed in learning bioassays in Drosophila. As rewards, we allowed our subjects to blood-feed or detect human breath, both representatives of natural cues to host presence. The recurrent failure to find unequivocal and strong evidence of associative learning under these settings leads us to cast serious doubts on the possibility that Ae. aegypti could take advantage of memorybased behaviour under natural conditions. We still cannot rule out the possibility that our negative results derive from a failure to present the individuals with the right context in which they could learn, or to present situations where subjects would be under the proper physiological or environmental conditions for making the relevant choices based on their previous experiences. Additionally, it is also possible that Type II statistical errors prevented the detection of learning effects of small magnitude in individual tests (Cohen 1988). Although this possibility cannot be ruled out, it is weakened by the frequent observation of results in the opposite direction as that predicted by the learning hypothesis, coupled to the recurrent failure to detect significant learning effects in many and different experiments. Finally, there is the possibility that the behavioural repertoire of the mosquitoes was not representative of that observed in nature, given the long history of laboratory life (Wood 1977). Unfortunately, by the time the present experiments were performed we were prevented from using individuals collected from nature due to the aggravation of dengue epidemics in several countries.

However, if alternatively, our results do prove to reflect a lack of learning capacity in Ae. aegypti, we can discuss why it would be so. It is interesting to note that Mwandawiro et al. (2000) found that the Culex species studied (Cx. tritaeniorhynchus, $C x$. vishnui and $C x$. gelidus) had an overall tendency to return to the host species upon which they had fed previously, but not $A e$. vexans. Are Aedes species therefore less able to learn? Regarding Ae. aegypti there are reports of movements of up to near $1 \mathrm{~km}$ (Reiter et al. 1995) but this species does not typically fly further than a few hundred meters, and most of its displacements are within few meters (Service 1997, Clements 1999). Resting and oviposition sites and feeding sources are usually near their hosts (Clements 1999), and when these resources are not available, mosquitoes seem simply to disperse, as was found with oviposition behaviour (Reiter et al 1995, Edman et al. 1998). Therefore, the presence of resources in a range of space where an individual can easily detect and identify them may not provide a strong selection pressure to develop and/or maintain learning abilities.

We could also speculate that learning in Ae. aegypti might not be relevant to assess the value of their resources on a long term (inter-gonotrophic cycle) basis, as odours associated with previous blood-feeds may not necessarily provide a more reliable and economical source of information about the host defensiveness than, for instance, the immediate evaluation made in each approach and probing stage. But, at least, as a diurnal host seeker in which the mortality risk associated with host defence is likely to be quite high, we would expect an avoidance behaviour toward individual hosts that show defensive behaviour (Gibson \& Torr 1999), and here some form of short-term learning could play an important role. Our vibration or electrical shock experiments do not suggest it, and they are in agreement with the findings of Canyon et al. (1998) in which defensive host behaviour did not necessarily lead to a change of human host by individual blood-seeking Ae. aegypti females.

\section{ACKNOWLEDGEMENTS}

To Luke Alphey for providing us with mosquitoes, Peter Burns and José Eduardo Bracco for technical assistance, Gordon Hamilton for advice on design of the olfactomenter, Tim Tully for the electric grid, Lester Wadhams for the pure citronella oil, and Cynthia Schuck-Paim, Armando Ulloa Garcia, Paul Johnson, Roger J Wood, Sarah E Randolph, Martin C Birch, Nicola Seal, Phillip J McCall, Steve J Simpson and three anonymous referees for helpful comments on the project and/ or manuscript.

\section{REFERENCES}

Arredondo-Jimenez JI, Bown DN, Rodriguez MH, Villarreal C, Loyola EG, Frederickson CE 1992. Tests for the existence of genetic determination or conditioning in host selection by Anopheles albimanus (Diptera, Culicidae). J Med Entomol 29: 894-897.

Canyon DV, Hii JLK, Muller R 1998. Multiple host-feeding and biting persistence of Aedes aegypti. Ann Trop Med Parasitol 92: 311-316.

Canyon DV, Hii JLK, Muller R 1999. The frequency of host biting and its effect on oviposition and survival in Aedes aegypti (Diptera:Culicidae). Bull Entomol Res 89: 35-39.

Cardew G 1996. Olfaction in Mosquito-host Interactions, Willey, Chichester, $331 \mathrm{pp}$.

Charlwood JD, Graves PM, Marshall TFD 1988. Evidence for a memorized home range in Anopheles farauti females from Papua New Guinea. Med Vet Entomol 2: 101-108.

Clements AN 1999. The Biology of Mosquitoes, Sensory Reception and Behaviour, Chapman \& Hall, London, 740 pp.

Cohen J 1988. Statistical Power Analysis for the Behavioural Sciences, Erlbaum, Hillsdale, 567 pp.

Dye C, Hasibeder G 1986. Population dynamics of mosquitoborne disease - Effects of flies which bite some people more frequently than others. Trans $R$ Soc Trop Med Hyg 80: 69-77.

Edman JD, Scott TW 1987. Host defensive behavior and the feeding success of mosquitoes. Insect Sci Appl 8: 617-622.

Edman JD, Scott TW, Costero A, Morrison AC, Harrington LC, Clark GG 1998. Aedes aegypti (Diptera:Culicidae) movement influenced by availability of oviposition sites. J Med Entomol 35: 578-583.

Ernst R, Heisenberg M 1999. The memory template in Drosophila pattern vision at the flight simulator. Vision Res 39 : 3920-3933.

Gibson G, Torr SJ 1999. Visual and olfactory responses of haematophagous Diptera to host stimuli. Med Vet Entomol 13: 2-23. 
Giglioli MEC 1965. The age composition of Anopheles melas Theobald (1903) populations collected simultaneously by different methods in The Gambia, West Africa. Cahiers ORSTOM 3: 11-26.

Gillies MT 1964. Selection for host preference in Anopheles gambiae. Nature 203: 852-854.

Hii JLK, Chew M, Sang VY, Munstermann LE, Tan SG, Panyim S, Yasothornsrikul S 1991. Population genetic-analysis of host seeking and resting behaviors in the malaria vector, Anopheles balabacensis (Diptera, Culicidae). JMed Entomol 28: 675-684.

Hurlbert SH 1984. Pseudoreplication and the design of ecological field experiments. Ecol Monogr 54: 187-211.

Jones JC, Pilitt DR 1973. Blood-feeding behavior of adult Aedes aegypti mosquitoes. Biol Bull 145: 127-139.

Kellogg FE, Wright RH 1962. The guidance of flying insects. V. Mosquito attraction. Can Entomol 94: 1009-1016.

Kelly DW 2001. Why are some people bitten more than others? Trends Parasitol 17: 578-581.

Kelly DW, Thompson CE. 2000. Epidemiology and optimal foraging: modelling the ideal free distribution of insect vectors. Parasitology 120: 319-327.

McCall PJ, Eaton G 2001. Olfactory memory in the mosquito Culex quinquefasciatus. Med Vet Entomol 15: 197-203.

McCall PJ, Kelly DW 2002. Learning and memory in disease vectors. Trends in Parasitol 18: 429-433.

McCall PJ, Mosha FW, Njunwa KJ, Sherlock K 2001. Evidence for memorized site-fidelity in Anopheles arabiensis. Trans R Soc Trop Med Hyg 95: 587-590.

Muir LE, Kay BH, Thorne MJ 1992a. Aedes aegypti (Diptera, Culicidae) vision - Response to stimuli from the optical environment. J Med Entomol 29: 445-450.

Muir LE, Thorne MJ, Kay BH 1992b. Aedes aegypti (Diptera, Culicidae) vision - Spectral sensitivity and other percep- tual parameters of the female eye. J Med Entomol 29: 278281.

Mwandawiro C, Boots M, Tuno N, Suwonkerd W, Tsuda Y, Takagi M 2000. Heterogeneity in the host preference of Japanese encephalitis vectors in Chiang Mai, Northern Thailand. Trans R Soc Trop Med Hyg 94: 238-242.

Quinn WG, Harris WA, Benzer S 1974. Conditioned behavior in Drosophila melanogaster. Proc Natl Acad Sci USA 71: 708-712.

Rawlings P, Curtis CF 1982. Tests for the existence of genetical variability in the tendency of Anopheles culicifacies species $\mathrm{B}$ to rest in houses and to bite man. Bull WHO 60: 427-432.

Reiter P, Amador MA, Anderson RA, Clark GG 1995. Dispersal of Aedes aegypti in an urban area after blood-feeding as demonstrated by rubidium-marked eggs. Am J Trop Med Hyg 52: 177-179.

Renshaw M, Service MW, Birley MH 1994. Host finding, feeding patterns and evidence for a memorized home-range of the mosquito Aedes cantans. Med Vet Entomol 8: 187-193.

Ribbands CR 1949. Studies on the attractiveness of human populations to anophelines. Bull Entomol Res 40: 227-238.

Service MW 1997. Mosquito (Diptera : Culicidae) dispersal The long and short of it. J Med Entomol 34: 579-588.

Sokal RR, Rohlf FJ 1995. Biometry, WH Freeman \& Co., New York, $887 \mathrm{pp}$.

Tully T, Quinn WG. 1985. Classical-conditioning and retention in normal and mutant Drosophila melanogaster. J Comp Physiol A-Sens Neural Behav Physiol 157: 263-277.

Waddell S, Quinn WG 2001. Flies, genes, and learning. Annu Rev Neurosci 24: 1283-1309.

Wood RJ 1977. Genetic aspects of "quality" in mass reared insects. In Association Euratom-ITAL, The Use of Ionizing Radiation in Agriculture, Wageningen, The Netherlands, p. 301-309. 